\title{
ECHOPHYSICS
}

\section{The first European Centre for the History of Physics in Poellau (Austria)}

\author{
- Hartmut Kahlert ${ }^{1}$, Heinz Krenn ${ }^{2}$ and Lily Wilmes ${ }^{3}$ - DOI: 10.1051/epn/2011404 \\ 口 ${ }^{1}$ Emeritus Prof. Dr. Hartmut KAHLERT, former Rector of the Graz University of Technology - kahlert@tugraz.at \\ - ${ }^{2}$ Prof. Dr. Heinz KRENN, Institute for Experimental Physics at the Graz Karl-Franzens-University - heinz.krenn@kfunigraz.ac.at \\ [ ${ }^{3}$ Dr. Lily WILMES, The Victor Francis Hess Society, Poellau - info@victorfhess.org
}

\section{In 2010, the Austrian scientific community celebrated the foundation of the Vienna Institute of Radium Research in 1910, then affiliated with the former Imperial and Royal Academy of Sciences of the Austro-Hungarian Monarchy. In 2012, another centennial event will take place: the 100th anniversary of the discovery of cosmic rays by the Austrian physicist Victor F. HESS, who was awarded the Nobel Prize for Physics in 1936.}

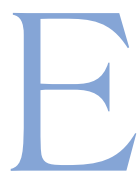

CHOPHYSICS combines the complete heritage exhibits of both these commemorative events. It was the author and physicist Peter Maria SCHUSTER ${ }^{*}$, who, besides his concern for the rescue and conservation of historically valuable experimental physics equipment, foresaw the two upcoming centennials of world-wide scientific interest, founded the Victor F. Hess Society in 2007, and established the first European Centre for the History of Physics in 2008. Both institutions are hosted in a former Augustinian Canonry of Stift Poellau in Styria, Austria.

\section{The ECHOPHYSICS initiative}

To facilitate the public access to the records about the early research on radioactivity, on the earth-bound nuclear and on the cosmic radiation, and to the first complete heritage of Victor Francis Hess, has needed first to rescue and gather the original instruments, specimens and paraphernalia he used with

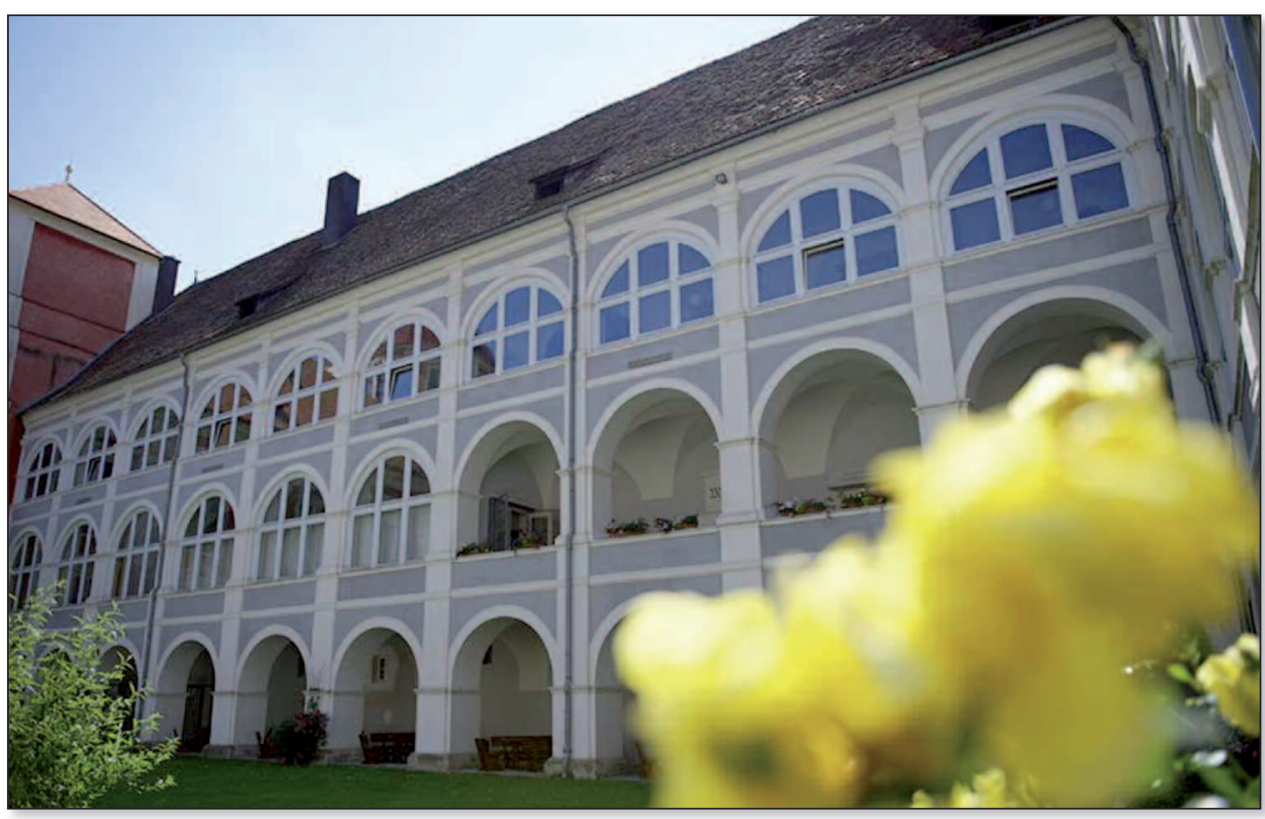

$\Delta$ The Poellau Centre other physicists. Furthermore, it was desirable to present the documentation in an appealing, spacious and secure location. The creation of the first permanent and bilingual

Note

* Dr. Peter Maria SCHUSTER is the initiator and director of ECHOPHYSICS and the president of the Victor Francis Hess Society; Dr. SCHUSTER is Chair of the EPS/History of Physics Group; pm.schuster@echophysics.org exhibition "Radiation and Mankind" (Strahlung, der ausgesetzte Mensch) followed, the Grand Opening of which taking place in May 2010 at the first European Centre for the History of Physics (ECHOPHYSICS) and the related Victor F. Hess Research and Heritage Centre at Poellau Castle in Styria, Austria. This site between Vienna and Graz is within a one-hour drive from 
both cities. The material on display comes mostly from Austrian universities (Vienna, Innsbruck and TU Graz) and the Austrian Academy of Sciences. Some comes from private collectors, but also from interested companies like Carl ZEISS. Without the unremitting engagement of a quite large team of physicists, most of whom are emeritus professors, from Austria and abroad, who set up the documentation and sorted and took care of the instruments to be shown, the task to set up ECHOPHYSICS and the exhibition could not have come true.

\section{The objective of ECHOPHYSICS}

In a novel approach, ECHOPHYSICS aims at intensifying the awareness of the scientific history of physics: the exhibition "Radiation and Mankind" is accompanied by meetings of physicists and historians of physics interested in the emergence of discovery. In 2010, ECHOPHYSICS and the Victor F. Hess Society hosted two international conferences in Poellau: "The Roots of Physics in Europe" (May 28 - 30) and the "Georg von Peuerbach Symposion" (October 8 - 9): "Models of the Real World-from the Late Middle Ages until the Age of Enlightenment".

These are examples of activities planned to accomplish the organizers ambition to raise the awareness for the pioneering discoveries in Late Habsburg Austria particularly in the field of radiation physics. The instrumentation and related pieces of evidence had long been hidden and, as a long term goal, their importance as crucial contributions to the emergence of Modern Physics in Europe needs to be shown.

\section{Touring "Radiation and Mankind", the first exhibi- tion at ECHOPHYSICS}

More than 80 illustrated bilingual text panels, a rich collection of original documents and a mesmerizing

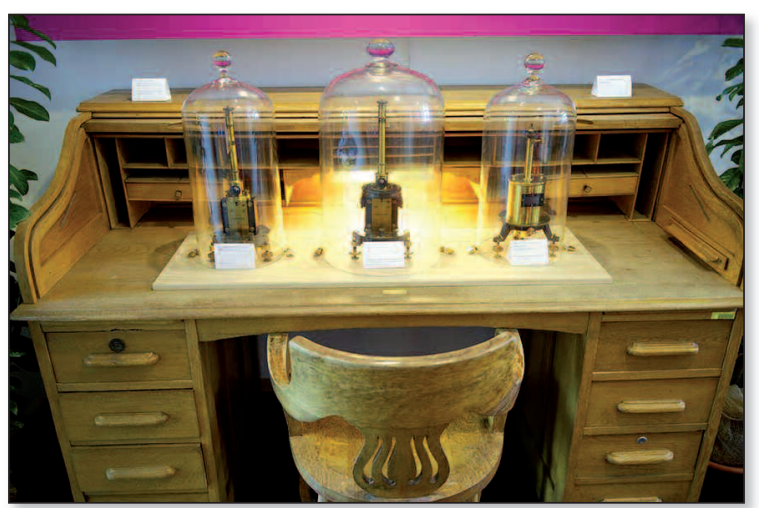

$\Delta$ Viktor F. Hess desk

set of nearly 600 valuable historical instruments accompany the visitor along the path through a sequence of nine exposition halls or sections, each being put in a vivid colour from the visible spectrum thus escorting the spectator through the conceptual evolution of physics general notion of radiation. The exhibition "Radiation and Mankind" reopens in Poellau Castle on the $7^{\text {th }}$ of May, 2011.

The large entrance hall displays the over-life-sized portraits of the famous Austrian physicists, who are related to radiation physics: Christian Doppler, Joseph Loschmidt, Josef Stefan and Ludwig Boltzmann. Besides, the explaining panels and showcases inform about their biography and scientific career, including most valuable historical documents. A caloric motor designed and patented by Loschmidt in 1868 and the original instruments used by Stefan to prove his $\mathrm{T}^{4}$ law for the radiation of heat are on exhibit.

From light and sound propagation (Doppler) to the bricks and building schemes of matter (Loschmidt), from radiation of heat (Stefan) through energy conversion (Boltzmann) and ionisation plus static electricity, the way to radioactivity is well documented.

A photomontage of the entrance portal to the Vienna Institut für Radiumforschung invites the visitor to view the rich historical heritage - provided by the Vienna University - of this famous 'Radium Institute' as it was commonly named, which had been established with the financial aid of Carl Kupelwieser in 1910, before the Institut Curie in Paris. The 'Radium Institute' benefited from the rich uranium ores mined from deposits at Joachimsthal (today Jáchimov in the Czech Republic), which made it, besides Paris, Berlin and Manchester, an early and internationally renowned centre for the radium research.

Some luminescent uranium-rich minerals can be seen at this section of the exhibition as well as the greatest part of the original instruments of the 'Radium Institute'. The original office desks and chairs of both Stefan Meyer and Victor F. Hess, can be admired. This section also includes detailed information on the exploration and discovery of the ionising particles in the atmosphere - a phenomenon named cosmic radiation by its discoverer Victor F. Hess.

The difficult access of the lay public to the section on mechanical waves and shock-waves is being smoothened by a hands-on facility in the upcoming 2011 season, where also a state-of-the-art information on research on, and applications of, shock-waves, with a related early experimental set-up according to Ernst Mach, is shown.

The panels and exhibits of the next hall describe the historical evolution of the two distinct phenomena: electricity and magnetism and how the exploration of these twin effects had led to the unified theory of electromagnetism. The shelves of a multitude of huge glass boards bear the rich collection of the various relevant historical apparatus - including an attractive replication of Guglielmo Marconi's wireless transmission of radio-waves using a spark-discharge and a 'fritter' as receiver.

From electromagnetism to light radiation, eye-catching crystals explain the physics behind the visible colours and the refraction, diffraction and fluorescence effects. 
Viewing the minerals under UV exposure or by a microscope gives repeatedly rise to ravishing exclamations by the spectators. 'Handling' light radiation and its effects requests the application of optics to produce a variety of optical components and to assemble these into measuring equipment: the largest hall with its baroque stucco and fresco decorated ceiling is dedicated to this subject and shows the entire range of historical optical instruments.

Emphasis is also given to medical application in ophthalmology and gynaecology.

Taking up again the Doppler Principle from the very beginning of the exhibition tour leads to the next hall, which is devoted to exploring the space beyond the earth's atmosphere and to understanding our cosmos. Panel texts and impressive illustrations explain satellite laser ranging, satellite telescoping with the CoRot spacecraft aiming at the study of star-quakes and at the search for exo-planets. One learns also about the cosmic rays and the implications of cosmic microwave background radiation-opening our eyes for the evolution of the universe from the Big Bang on.

The explanations and illustrations about the currently largest accelerator system LHC at CERN with the purpose of studying the basic constituents and the structure of matter might remind the attentive visitor of how Loschmidt had dealt with a similar question although at an almost tangible earth-bound level. Furthermore, the Atominstitut, the 'Vienna Institute of Atomic and Subatomic Physics', founded in 1958 is presented with its early realization of a neutron interferometer, which showed first the wave nature of neutrons in 1974.

The non-specialist may be happy to find a concluding dual panel on the risks of X-ray and nuclear radiation and on the risks of microwaves, radio waves, radar and infrared to

soothe his tantalizing questions about the risks of the gigahertz radiation from his mobile phone.

\section{The Victor F. Hess Research and Heritage Centre}

The 'Victor F. Hess Research and Heritage Centre', associated to ECHOPHYSICS, shows the inheritance, both of the physicist and of the private person Hess. This collection makes an invaluable part of "Radiation and Mankind". All the exhibits devoted to Victor F. Hess have thematically been integrated into the main topic of 'Radiation'. They include on the one hand lent by the Vienna University the scientific instruments and the original furniture from his stay at the Vienna Institut für Radiumforschung, where Hess had worked out the data from his decisive balloon ride in 1912, and, on the other hand, his entire private heritage (medals and certificates of awards, photos, notes, letters, including also the original Nobel Prize Certificate) generously let by Victor's step-grandsons Arthur and William Breisky at the Poellau based 'Victor F. Hess Society' since May 2010. Moreover, an original hot-air balloon from the Vienna Arsenal illustrates how the courageous young Victor F. Hess rode up to 5.350 metres and discovered that

$\checkmark$ Hess Nobel Prize on the Balloon nacelle used for cosmic ray detection

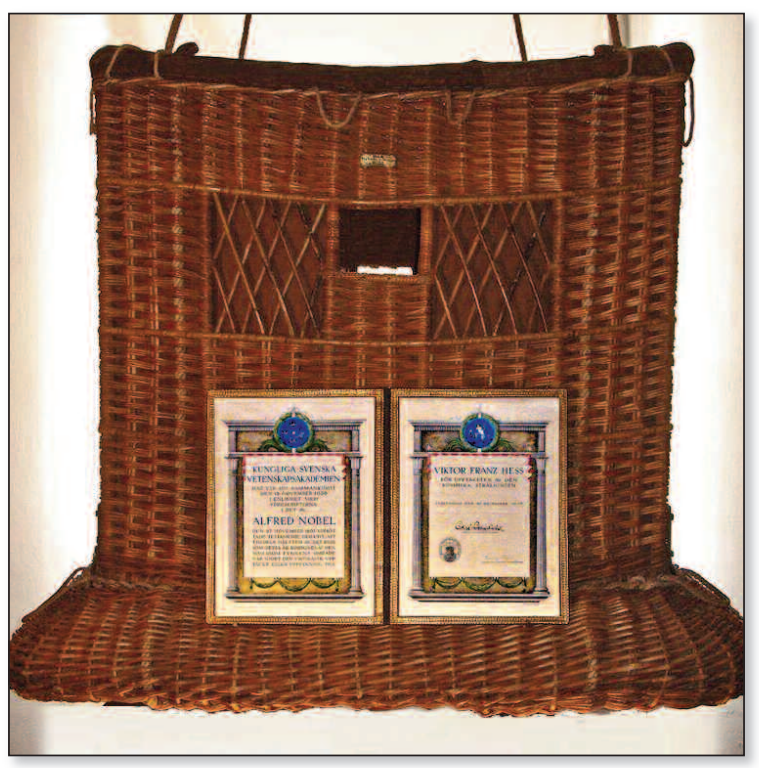

the ionising or cosmic radiation is of extraterrestrial origin. A last panel displays a warm though posthumous welcome back home to Austria for Victor F. Hess, who, dispensed of his professorship at Graz University by the Nazi regime in 1938, had lived in exile in the USA during World War II and continued working at Fordham University, NY, since then.

"Radiation and Mankind" reveals the links between the pioneering discoveries in the field and the modern research on hard cosmic rays (beyond $10^{15} \mathrm{eV}$ ). Thus a viewing of the Namibia experiment, called "H.E.S.S."-High Energy Stereoscopic System-informs the visitor that, in the present day gamma-astronomy, the registered H.E.S.S. signals are understood as a fingerprint of the hard primary cosmic rays.

In 2012, the centennial of the discovery of cosmic rays by Victor F. Hess will be celebrated throughout the world. An international conference for and by physicists that centres on the related historical research will be held at the European Centre for the History of Physics ECHOPHYSICS in Poellau. This Poellau ECHOPHYSICS conference is only one of a series of international specialists conferences on this topic that will be held in Vienna and Innsbruck, in Denver, Moscow and in Bad Saarow/Pieskow near Berlin. It is planned to take place in May/June 2012, after the starting conferences in Vienna and Innsbruck. 